\title{
Nicotinamide Phosphoribosyltransferase in Rheumatoid Arthritis
}

\author{
Daniel P. Heruth ${ }^{1}$, Li Qin Zhang ${ }^{1}$ and Shui Qing Ye ${ }^{2 *}$
}

${ }^{1}$ Department of Biomedical and Health Informatics, Children's Mercy Hospitals and Clinics, University of Missouri School of Medicine, Kansas City, MO 64108, USA

${ }^{2}$ Department of Pediatrics, Children's Mercy Hospitals and Clinics, University of Missouri School of Medicine, Kansas City, MO 64108, USA

\begin{abstract}
Nicotinamide Phosphoribosyltransferase (Nampt) is a pleiotropic protein with multiple functions, including catalyzing a rate-limiting reaction of nicotinamide adenine dinucleotide (NAD) synthesis in a mammalian salvage pathway and mediating the innate immune system's inflammatory response. The enzymatic activity of Nampt has been well characterized; however, the mechanism(s) by which Nampt regulates cytokine signaling have not been fully elucidated. Rheumatoid arthritis (RA) a chronic, systemic autoimmune disorder in which the pathological roles of proinflammatory cytokines such as Tumor Necrosis Factor (TNF), interleukin (IL)-1 $\beta$, and IL- 6 have been clearly demonstrated. Recently, studies have shown that serum and synovial levels of Nampt are elevated in both RA patients and in mice with collagen induced arthritis (CIA), and that inhibition of Nampt activity decreases the expression of TNF, IL-1 $\beta$, and IL-6. Thus, Nampt may represent a potential new therapeutic target in RA. This review will focus on Nampt's role in NAD metabolism and cytokine signaling in the context of the pathophysiology and therapeutic potential of RA.
\end{abstract}

Keywords: Nampt; PBEF; Visfatin; Rheumatoid arthritis; Cytokine

\section{Introduction}

Rheumatoid arthritis [RA] is a chronic, systemic autoimmune disorder which affects $1 \%$ of the world's population, develops most commonly in adults between 40 - 70 years old, and occurs more frequently in women than in men [1,2]. Although the etiology of the disease has not been fully elucidated, the pathogenesis of RA is characterized by the influx of cells from both the innate and the adaptive immune systems [3-5]. These infiltrating cells induce increased pro-inflammatory cytokine production, decreased synthesis of anti-inflammatory cytokines, and the subsequent activation and proliferation of synovial tissue. The severe imbalance in the cytokine signaling pathways ultimately lead to the thickening and progressive destruction of joint membrane, cartilage and bone [2-5].

Characterization of the cytokine signaling pathways involved in RA has provided a significant opportunity for identifying pro-inflammatory cytokines which can be targeted for novel therapeutic intervention. Biological response modifiers, such as antagonists of interleukin-1 (IL1) (anakinra) and tumor necrosis factor (TNF) (etanercept, infliximab, and adalimumab), have led to major advances in RA therapy $[2,5,6]$. Clinical improvements of the inflammatory symptoms have been achieved, especially when the biological therapies are administered in combination with methotrexate, a traditional disease-modifying antirheumatic drug (DMARD) [2,7]. Naturally, the therapeutic success of both IL-1 and TNF antagonists has led to the identification and characterization of additional proteins for target development $[5,6]$. One such target is Nicotinamide Phosphoribosyltransferase (Nampt), [8-13] a highly conserved protein that is essential for development, as a homozygous knockout of the Nampt gene in mice is embryonically lethal. Three biological functions of Nampt have been characterized [13]. Nampt catalyzes the initial reaction in the enzymatic conversion of nicotinamide adenine dinucleotide (NAD) from nicotinamide (NAM) in a mammalian salvage pathway. Nampt also functions as an extracellular cytokine/growth factor initially termed Pre-B Cell Colony Enhancing Factor (PBEF). And finally, Nampt has been characterized as visfatin, an adiopocyte-derived adipokine that induces insulinmimetic effects. Although, these three functions appear to be distinct, they are all intricately associated with the transduction of physiological signals.
Nampt expression is up-regulated in a variety of acute and chronic inflammatory diseases including acute lung injury [14], sepsis [15], inflammatory bowel disease, [16,17] atherosclerosis, [18,19] osteoarthritis [20-22] and rheumatoid arthritis [17,23-30]. Recent findings have demonstrated that targeted inhibition of Nampt attenuates the inflammatory process and disease progression in mouse models of arthritis [31,32], suggesting that Nampt plays a key role in the pathogenesis of RA. However, determining the mechanism(s) by which Nampt regulates the inflammatory response in RA is both challenging and intriguing because of Nampt's pleiotropic molecular functions $[8,11,13,33]$ Nampt is localized within the nucleus and cytoplasm, however, it is also actively secreted from cells. The intracellular (iNampt) and extracellular (eNampt) forms function as dimeric type II phosphoribiosyltransferases in NAD biosynthesis [34,35]. NAD is an important co-factor for NAD consuming enzymes involved in DNA repair, apoptosis, metabolism, immune reactions, and inflammatory responses, and several studies have demonstrated that Nampt's enzyme activity is required for its cytokine activity [31,32,34]. However, two recent studies utilizing mutationally inactivated Nampt have demonstrated that the enzymatic activity of eNampt was not necessary for the cytokine-like cell signaling properties [36,37]. These studies suggest that eNampt must either directly or indirectly induce a receptor-mediated signal transduction cascade to activate the cytokine network. This review will address the role of Nampt in NAD metabolism and cytokine signaling in the context of the pathophysiology and therapeutic potential of RA.

\section{The molecular functions of nampt}

The use of three names, PBEF, visfatin, and Nampt to describe the

*Corresponding author: Shui Qing Ye, Departments of Pediatrics, Children's Mercy Hospitals and Clinics, University of Missouri-School of Medicine, 2401 Gillham Road, PRC/4th FL, Kansas City, MO 64108, E-mail: yes@umkc.edu

Received May 04, 2011; Accepted June 15, 2011; Published June 23, 2011

Citation: Heruth DP, Zhang LQ, Ye SQ (2011) Nicotinamide Phosphoribosyltransferase in Rheumatoid Arthritis. J Bioanal Biomed 3: 076-080. doi:10.4172/1948-593X.1000047

Copyright: ( 2011 Heruth DP, et al. This is an open-access article distributed under the terms of the Creative Commons Attribution License, which permits unrestricted use, distribution, and reproduction in any medium, provided the original author and source are credited. 
molecular functions of one protein reflects upon the rich history of their discoveries. Recently, Nampt was approved as the official name of this gene by the Human Genome Organization Gene Nomenclature Committee, therefore, we will use Nampt throughout this review.

Samal et al. [38] first identified Nampt as pre-B-cell colonyenhancing factor (PBEF), a potential growth factor expressed and secreted by activated human peripheral blood lymphocytes based upon its ability to stimulate early stage $\mathrm{B}$ cell maturation in conjunction with IL-7 and stem cell factor (SCF). The Nampt cDNA was cloned using a degenerate oligonucleotide probe, designed on the basis of the similarity of the signal peptidase processing site within the coding sequences of five different cytokines, GM-CSF, IL-1 $\beta$, IL-2, IL- 6 and IL-13 [38]. Surprisingly, analysis of the Nampt cDNA revealed that neither the DNA nor the protein sequence shared any homology to other known cytokines, however, within the 3' untranslated sequence of Nampt cDNA there were multiple TATT motifs [38], a hallmark feature of cytokines. Although Nampt was secreted by activated lymphocytes, it lacked the canonical signal peptide sequence typically found in secreted cytokines.

Martin et al. [39] demonstrated that the bacterial gene, NadV, which shared sequence homology to Nampt, had nicotinamide phosphoribosyltransferase activity. Subsequently, Rongvaux et al. [40] verified that the mammalian protein also possesses nicotinamide phosphoribosyltransferase activity. In mammalian cells, the enzymes nicotinamide phosphoribosyltransferase (Nampt) and nicotinamide mononucleotide adenylyltransferase (Nmnat) constitute an NAD salvage pathway. Nampt catalyzes the condensation of nicotinamide with 5-phos-phoribosyl 1-pyrophosphate (PRPP) to yield nicotinamide mononucleotide (NMN), which is then converted into NAD by NAMPT. Revollo and colleagues demonstrated that Nampt is the rate-limiting enzyme in the salvage pathway of mammalian NAD biosynthesis [41].

Fukuhara et al. [42] identified an adipokine secreted by visceral fat cells, termed visfatin, which increased both glucose uptake and the synthesis of triglycerides. They also proposed that visfatin induced its insulin-mimetic effects by binding to the insulin receptor (IR). However, subsequent studies have not been able to verify that Nampt is capable of binding the IR, and consequently the claim of a Nampt-IR interaction has been retracted. Further evidence against a Nampt-IR interaction was provided by Revollo and colleagues, who demonstrated that Nampt regulates insulin secretion in pancreatic beta cells as a systemic NAD biosynthetic enzyme [34].

Nampt has two forms, an intracellular form (iNampt) and a secreted, extracellular form (eNampt). It has been well documented that iNampt is a nicotinamide phosphoribosyltransferase, and that its enzymatic activity is attributed for its intracellular functions. However, the mechanism(s) by which eNampt completes its various functions have not been elucidated fully. In the study by Revollo et al. [34] the nicotinamide phosphoribosyltransferase activity of eNampt is necessary for the regulation of insulin secretion in pancreatic beta cells. However, two recent studies utilizing mutationally inactivated Nampt have demonstrated that the enzymatic activity of eNampt was not necessary for the cytokine-like cell signaling properties. Li et al. [36] reported that eNampt protected macrophages from endoplasmic reticulum stress-induced apoptosis by activating an IL-6/STAT3 signaling pathway via a nonenzymatic mechanism. Liu and colleagues provided the first evidence that Nampt can regulate the expression of the inflammatory cytokines IL-8, IL-16, and CCR3 via a nonenzymatic function which is activator protein $1(\mathrm{AP}-1)$ dependent, in part via the p38 mitogen-activated protein kinase (p38 MAP) and Jun N-terminal kinases (JNK) pathways [37].

\section{Epidemiological surveys of nampt expression in rheumatoid arthritis}

Otero et al. [23] first reported that patients with rheumatoid arthritis showed higher plasma levels of Nampt, leptin, and adiponectin than healthy controls. The authors proposed that these adipokines could coordinate the inflammatory process in RA, and at the very least could serve as biomarkers for the disease. These findings were confirmed by Nowell et al. [30] Bretano et al. [24] and Matsui et al. [25] Nowell and colleagues detected elevated levels of Nampt in synovial fluid from RA patients when compared with osteoarthritis (OA) patients [30] Nampt expression was immunolocalized within the synovial lymphoid aggregates, which consisted of B cells, T cells, dendritic cells, and plasma cells, as well as within several additional cell types associated with the pathophysiology of RA, including endothelial cells, fibroblast-like synoviocytes (FLS), macrophage-like synoviocytes (MLS), and adipocyte-like cells [30]. Bretano et al. [24] also demonstrated that Nampt expression was elevated in the joints of RA patients, especially in the FLS localized at the points of invasion into the synovial lining and cartilage. Matsui et al. [25] detected elevated Nampt mRNA expression in synovial tissue, peripheral blood mononuclear cells (PBMCs), and peripheral blood granulocytes (PBGs) isolated from RA patients compared to healthy controls. Subsequent surveys [17,26-29] have verified that expression of Nampt in serum, synovial fluid, and synovial tissue is elevated in patients with RA. In a large survey of 167 RA patients and 91 control subjects by Rho et al. [26] elevated levels of Nampt correlated with both radiological joint destruction and mediators of inflammation. A study by StraburzynskaLupa and colleagues, found that serum levels of Nampt were lower in female RA patients with abdominal obesity than in female RA patients without abdominal obesity [29]. Furthermore, elevated serum levels of Nampt did not correlate with either the degree of insulin resistance [28,29] or body mass index (BMI) [26,43]. Mesko et al. [17] measured mRNA expression in PBMCs isolated in patients with inflammatory bowel disease (IBD), psoriasis, and RA. They identified five genes (ADM, AQP9, CXCL2, IL10 and NAMPT) that differentiate between patients with chronic inflammation and healthy controls, further suggesting that RA shares common pathogenic mechanisms with other inflammatory diseases.

\section{Pathogenic mechanisms of nampt in rheumatoid arthritis}

Nampt is a pleiotropic protein which can induce the expression of a number of genes (e.g. CCR2, CCR3, Cox-2, IL-6, IL-8, IL-16, ICAM1, MCP-1, MMP-2, MMP-9, VCAM1, VEGF) [44-52] and mediate the activities of several pathways (e.g. ERK 1/ERK2, [50,52,53] IL6/STAT3, [36] p38 MAPK, [52] PI3K/Akt [48,49,52]). Analyses of RA diseased tissues support the role of Nampt as an effective mediator of cytokine activity. Bretano et al. [24] linked Nampt activity with inflammatory cytokine cascades by stimulating FLS from RA patients with recombinant human Nampt. Nampt activated the transcription factors $\mathrm{NF}-\mathrm{kB}$ and $\mathrm{AP}-1$, and induced the expression of the metalloproteinases MMP-1 and MMP-3, and the cytokines IL-6, IL-8. Expression of IL-6 and TNF was also induced in primary blood monocytes treated with recombinant human Nampt. These observations were verified by siRNA knockdown of Nampt in FLS which significantly inhibited basal and TLR ligand-induced production of IL-6, IL-8, MMP-1, and MMP-3. Nowell et al. [30] also reported that STAT-3 dependent IL-6 trans-signaling regulates the expression of Nampt in human FLS and in an antigen-induced mouse model of arthritis (AIA). These findings 
support that Nampt affects the innate immune system's inflammatory response. However, the mechanism by which Nampt mediates the cytokine signaling cascade has not yet been determined.

The findings that Nampt can both induce the expression of cytokines, like IL-1 $\beta$, IL- 6 and TNF, but also be induced by these same cytokines, suggest that these signaling pathways must be intricate networks with both intracellular and extracellular components. The enzymatic activity of both iNampt and eNampt, suggests that NAD signaling is a critical component of these complex cytokine networks. NAD functions as both an electron carrier for redox reactions in metabolism and as a substrate for NAD-consuming enzymes. NAD is consumed as a donor of ADP-ribose, with the concomitant release of nicotinamide (NAM) as a byproduct. Therefore, the salvage pathway of NAD synthesis is critical for maintaining the functions of a wide variety of NAD-dependent enzymes in the cytoplasm and nucleus. The NAD-consuming enzymes, which have multiple roles in cellular regulation, including gene silencing, DNA repair, apoptosis, aging, stress response, cell differentiation, immune reactions, and inflammatory responses, can be classified into three large protein families; the mono-ADP ribosyl transferases (ARTs) [54,55], the polyADP ribosyltransferases (PARPs) [54,56], and the sirtuins [54,57]. A member of the PARP family, PARP-1, functions as a co-activator of $\mathrm{NF}-\mathrm{kB}$ during the pathogenesis of inflammatory disorders, including RA [58] Gonzalez-Rey and colleagues showed that inhibition of PARP1 with the inhibitor 5-aminoisoquinolinone (AIQ) effectively reduced both the autoimmune and inflammatory responses in CIA mice, thus linking further the NAD signaling network to the pathogenesis of RA [59] Sirt1, a member of the sirtuin family, is a highly conserved NADdependent protein deacetylase and a key regulator of metabolic activity [60] While Sirt1 is positively regulated by NAD, NAM [61] and NADH [62] inhibit Sirt1 activity. Sirt1 regulates the inflammatory response by inactivating NF- $k \mathrm{~B}$, however, elevated levels of TNF induce cathepsin B cleavage of Sirt1, thus allowing further progression of the inflammatory cascade [63]. Further evidence supporting the importance of the NAD salvage pathway, is the finding that NAM inhibits the production of several pro-inflammatory mediators [64,65], including TNF, suggesting a complex regulatory feedback loop involving Nampt enzyme activity.

However, the discovery that enzymatically inactive eNampt can stimulate cytokine-like cell signaling, strongly suggests that an alternative signaling pathway exists $[36,37]$. The most likely explanation of these conflicting results is that Nampt regulates cytokine expression by a dual approach of controlling the synthesis of NAD, which serves as a signal molecule, and by directly or indirectly inducing a receptormediated signal transduction cascade to activate the cytokine network. However, to verify this hypothesis, it will be necessary to characterize further NAD's role in regulating cytokine expression, and to identify the potential ligands and receptors associated with transmitting the cytokine signal.

Cumulatively, these findings support the concept that the normal feedback loops for regulating NAD signaling and cytokine networks are dysregulated in RA, thus resulting in an amplified cytokine cascade with increased inflammation and tissue destruction.

\section{Therapeutic potential of regulating nampt expression in rheumatoid arthritis}

The dysregulation of Nampt activity in RA makes it an attractive target for therapeutic intervention. Evidence for this concept was established by Busso et al. [31] and Evans et al. [32] who demonstrated that Nampt is a key player in inflammatory arthritis. Collagen-induced arthritis (CIA) in mice is accompanied by increased expression of Nampt in both serum and in the arthritic paw. Administration of APO866, a specific competitive inhibitor of Nampt, effectively reduced the severity and progression of arthritis [31,32] with comparable activity to the TNF inhibitor, etanercept [31]. APO866 treatment also reduced the intracellular NAD concentration in inflammatory cells and circulating TNF levels in mice stimulated with lipopolysaccharide (LPS). In addition, the effect of APO866 was reversed by supplementation with NMN, the product of the NAMPTcatalyzed reaction [31]. In the study by Evans et al. [32] in vitro analysis of APO866 inhibition of Nampt performed in the human fibroblast cell line (HFFF2) demonstrated that Nampt regulates the expression of both cytokines and metalloproteinases (MMPs). APO866 was highly specific to Nampt and had no significant effect on the expression of the tissue inhibitor of metalloproteinase (TIMP)-1, [32] suggesting that the absence of TIMP-1 control of NAMPT-induced MMPs may contribute to an imbalance towards cartilage catabolism in arthritis. In vivo studies in CIA mice also demonstrated that the inhibition of Nampt activity prior to the clinical onset of arthritis correlated with the active suppression of cytokine and metalloproteinase activity, resulting in reduced inflammation and destruction in and around the synovial joint. ${ }^{32}$ These findings further link Nampt and NAD metabolism to inflammatory cytokine signaling and demonstrate that Nampt may be a potential therapeutic target in RA.

\section{Conclusion}

The correlation of elevated expression of Nampt with inflammation and tissue destruction has identified Nampt as an important mediator of the innate immune response and a potential target for RA therapy. Although the enzymatic activity of Nampt has been well characterized, additional research is needed to elucidate the mechanism(s) by which Nampt regulates both NAD metabolism and cytokine signaling. Inhibition of Nampt activity by APO866 in CIA mice has provided strong evidence that Nampt is a promising therapeutic target. However, screening for additional inhibitory molecules of Nampt is needed, as thrombocytopenia is a potential side effect of APO866 treatment in humans. Investigation of alternative therapies, like viral delivery of Nampt-sh RNA and anti-Nampt monoclonal antibody treatment should be also be initiated. Furthering these lines of study may contribute to mechanistic insights into the molecular pathogenesis of RA and new therapeutic strategies for RA, and inflammatory diseases in general.

\section{References}

1. Lee DM, Weinblatt ME (2001) Rheumatoid arthritis. Lancet 358: 903-911.

2. Scott DL, Wolfe F, Huizinga TW (2010) Rheumatoid arthritis. Lancet 376: 10941108 .

3. Cooles FA, Isaacs JD (2011) Pathophysiology of rheumatoid arthritis. Curr Opin Rheumatol 23: 233-240.

4. Gierut A, Perlman H, Pope RM (2010) Innate immunity and rheumatoid arthritis Rheum Dis Clin North Am 36: 271-296.

5. Firestein GS (2003) Evolving concepts of rheumatoid arthritis. Nature 423: 356361.

6. Buch MH, Emery $\mathrm{P}$ (2011) New therapies in the management of rheumatoid arthritis. Curr Opin Rheumatol 23: 245-251.

7. Allaart CF, Huizinga TW (2011) Treatment strategies in recent onset rheumatoid arthritis. Curr Opin Rheumatol 23: 241-244.

8. Luk T, Malam Z, Marshall JC (2008) Pre-B cell colony-enhancing factor (PBEF) visfatin: a novel mediator of innate immunity. J Leukoc Biol 83: 804-816. 
Citation: Heruth DP, Zhang LQ, Ye SQ (2011) Nicotinamide Phosphoribosyltransferase in Rheumatoid Arthritis. J Bioanal Biomed 3: 076-080. doi:10.4172/1948-593X.1000047

9. Imai S (2009) Nicotinamide phosphoribosyltransferase (Nampt): a link between NAD biology, metabolism, and diseases. Curr Pharm Des 15: 20-28.

10. Bao JP, Chen WP, Wu LD (2009) Visfatin: a potential therapeutic target for rheumatoid arthritis. J Int Med Res 37: 1655-1661.

11. Moschen AR, Gerner RR, Tilg H (2010) Pre-B Cell Colony Enhancing Factor/ NAMPT/Visfatin in Inflammation and Obesity-Related Disorders. Curr Pharm Des 16: 1913-1920.

12. Neumann E, Frommer K, Vasile M, Muller-Ladner U (2011) Adipocytokines as driving forces in rheumatoid arthritis and related inflammatory diseases? Arthritis Rheum 63: 1159-1169.

13. Zhang LQ, Heruth DP, Ye SQ (2011) Nicotinamide Phosphoribosyltransferase in Human Diseases. J Bioanal Biomed 3: 013-025.

14. Ye SQ, Simon BA, Maloney JP, Zambelli-Weiner A, Gao L, et al. (2005) Pre-Bcell colony-enhancing factor as a potential novel biomarker in acute lung injury. Am J Respir Crit Care Med 171: 361-370.

15. Jia SH, Li Y, Parodo J, Kapus A, Fan L, et al. (2004) Pre-B cell colonyenhancing factor inhibits neutrophil apoptosis in experimental inflammation and clinical sepsis. J Clin Invest 113: 1318-1327.

16. Moschen AR, Kaser A, Enrich B, Mosheimer B, Theurl M, et al. (2007) Visfatin, an adipocytokine with proinflammatory and immunomodulating properties. $J$ Immunol 178: 1748-1758

17. Mesko B, Poliska S, Szegedi A, Szekanecz Z, Palatka K, et al. (2010) Peripheral blood gene expression patterns discriminate among chronic inflammatory diseases and healthy controls and identify novel targets. BMC Med Genomics 3: 15.

18. Dahl TB, Yndestad A, Skjelland M, Øie E, Dahl A, et al. (2007) Increased expression of visfatin in macrophages of human unstable carotid and coronary atherosclerosis: possible role in inflammation and plaque destabilization. Circulation 115: 972-980.

19. Liu SW, Qiao SB, Yuan JS, Liu DQ (2009) Association of plasma visfatin levels with inflammation, atherosclerosis and acute coronary syndromes (ACS) in humans. Clin Endocrinol (Oxf) 71: 202-207.

20. Gosset M, Berenbaum F, Salvat C, Sautet A, Pigenet A, et al. (2008) Crucial role of visfatin/pre-B cell colony-enhancing factor in matrix degradation and prostaglandin E2 synthesis in chondrocytes: possible influence on osteoarthritis. Arthritis Rheum 58: 1399-1409.

21. Gabay O, Hall DJ, Berenbaum F, Henrotin Y, Sanchez C (2008) Osteoarthritis and obesity: experimental models. Joint Bone Spine 75: 675-679.

22. Chen WP, Bao JP, Feng J, Hu PF, Shi ZL, et al. (2010) Increased serum concentrations of visfatin and its production by different joint tissues in patients with osteoarthritis. Clin Chem Lab Med 48: 1141-1145.

23. Otero M, Lago R, Gomez R, Lago F, Dieguez C, et al. (2006) Changes in plasma levels of fat-derived hormones adiponectin, leptin, resistin and visfatin in patients with rheumatoid arthritis. Ann Rheum Dis 65: 1198-1201.

24. Brentano F, Schorr O, Ospelt C, Stanczyk J, Gay RE, et al. (2007) Pre-B cell colony-enhancing factor/visfatin, a new marker of inflammation in rheumatoid arthritis with proinflammatory and matrix-degrading activities. Arthritis Rheum 56: $2829-2839$

25. Matsui H, Tsutsumi A, Sugihara M, Suzuki T, Iwanami K, et al. (2008) Visfatin (pre-B cell colony-enhancing factor) gene expression in patients with rheumatoid arthritis. Ann Rheum Dis Apr 67: 571-572.

26. Rho YH, Solus J, Sokka T, Oeser A, Chung CP, et al. (2009) Adipocytokines are associated with radiographic joint damage in rheumatoid arthritis. Arthritis Rheum 60: 1906-1914.

27. Fagerer N, Kullich W (2010) Adipocytokines in rheumatoid arthritis and obesity. Wien Med Wochenschr 160: 391-398.

28. Ozgen M, Koca SS, Aksoy K, Dagli N, Ustundag B, et al. (2010) Visfatin levels and intima-media thicknesses in rheumatic diseases. Clin Rheumatol 30: 757763.

29. Straburzyńska-Lupa A, Nowak A, Pilaczyńska-Szcześniak $Ł$, StraburzyńskaMigaj E, Romanowski W, et al. (2010) Visfatin, resistin, hsCRP and insulin resistance in relation to abdominal obesity in women with rheumatoid arthritis. Clin Exp Rheumatol 28: 19-24.
30. Nowell MA, Richards PJ, Fielding CA, Ognjanovic S, Topley N, et al. (2006) Regulation of pre-B cell colony-enhancing factor by STAT-3-dependent interleukin- 6 trans-signaling: implications in the pathogenesis of rheumatoid arthritis. Arthritis Rheum 54: 2084-2095.

31. Busso N, Karababa M, Nobile M, Rolaz A, Van Gool F, et al. (2008) Pharmacological inhibition of nicotinamide phosphoribosyltransferase/visfatin enzymatic activity identifies a new inflammatory pathway linked to NAD. PLoS One 3: e2267.

32. Evans L, Williams AS, Hayes AJ, Jones SA, Nowell M (2011) Selective inhibition of PBEF/Visfatin/NAMPT suppresses leukocyte infiltration and cartilage degradation. Arthritis Rheum

33. Imai S (2009) The NAD World: a new systemic regulatory network for metabolism and aging--Sirt1, systemic NAD biosynthesis, and their importance. Cell Biochem Biophys 53: 65-74.

34. Revollo JR, Körner A, Mills KF, Satoh A, Wang T, et al. (2007) Nampt/PBEF/ Visfatin regulates insulin secretion in beta cells as a systemic NAD biosynthetic enzyme. Cell Metab 6: 363-375.

35. Wang T, Zhang X, Bheda P, Revollo JR, Imai S, et al. (2006) Structure of Nampt/PBEF/visfatin, a mammalian NAD+ biosynthetic enzyme. Nat Struct Mo Biol 13: 661-662.

36. Li Y, Zhang Y, Dorweiler B, Cui D, Wang T, et al. (2008) Extracellular Nampt promotes macrophage survival via a nonenzymatic interleukin-6/STAT3 signaling mechanism. J Biol Chem 283: 34833-34843.

37. Liu P, Li H, Cepeda J, Xia Y, Kempf JA, et al. (2009) Regulation of inflammatory cytokine expression in pulmonary epithelial cells by pre-B-cell colony-enhancing factor via a nonenzymatic and AP-1-dependent mechanism. J Biol Chem 284: 27344-27351.

38. Samal B, Sun Y, Stearns G, Xie C, Suggs S, et al. (1994) Cloning and characterization of the cDNA encoding a novel human pre-B-cell colonyenhancing factor. Mol Cell Biol 14: 1431-1437.

39. Martin PR, Shea RJ, Mulks MH (2001) Identification of a plasmid-encoded gene from Haemophilus ducreyi which confers NAD independence. J Bacteriol 183: $1168-1174$

40. Rongvaux A, Shea RJ, Mulks MH, Gigot D, Urbain J, et al. (2002) Pre-Bcell colony-enhancing factor, whose expression is up-regulated in activated lymphocytes, is a nicotinamide phosphoribosyltransferase, a cytosolic enzyme involved in NAD biosynthesis. Eur J Immunol 32: 3225-3234.

41. Revollo JR, Grimm AA, Imai S (2004) The NAD biosynthesis pathway mediated by nicotinamide phosphoribosyltransferase regulates Sir2 activity in mammalian cells. J Biol Chem 279: 50754-50763.

42. Fukuhara A, Matsuda M, Nishizawa M, et al. (2005) Visfatin: a protein secreted by visceral fat that mimics the effects of insulin. Science 307: 426-430

43. Gonzalez-Gay MA, Vazquez-Rodriguez TR, Garcia-Unzueta MT, Berja A Miranda-Filloy JA, et al. (2010) Visfatin is not associated with inflammation or metabolic syndrome in patients with severe rheumatoid arthritis undergoing anti-TNF-alpha therapy. Clin Exp Rheumatol 28: 56-62.

44. Ognjanovic S, Bao S, Yamamoto SY, Garibay-Tupas J, Samal B, et al (2001) Genomic organization of the gene coding for human pre-B-cell colony enhancing factor and expression in human fetal membranes. J Mol Endocrinol 26: 107-117

45. Liu P, Li H, Cepeda J, Zhang LQ, Cui X, et al. (2009) Critical role of PBEF expression in pulmonary cell inflammation and permeability. Cell Biol Int 33 19-30.

46. Li H, Liu P, Cepeda J, Fang D, Easley RB, et al. (2008) Augmentation of Pulmonary Epithelial Cell IL-8 Expression and Permeability by Pre-B-cell Colony Enhancing Factor. J Inflamm (Lond) 5:15.

47. Adya R, Tan BK, Chen J, Randeva HS (2008) Nuclear factor-kappaB induction by visfatin in human vascular endothelial cells: its role in MMP-2/9 production and activation. Diabetes Care 31: 758-760.

48. Adya R, Tan BK, Chen J, Randeva HS (2009) Pre-B cell colony enhancing factor (PBEF)/visfatin induces secretion of MCP-1 in human endothelial cells: role in visfatin-induced angiogenesis. Atherosclerosis 205: 113-119.

49. Adya R, Tan BK, Punn A, Chen J, Randeva HS (2008) Visfatin induces human endothelial VEGF and MMP-2/9 production via MAPK and PI3K/Akt signalling pathways: novel insights into visfatin-induced angiogenesis. Cardiovasc Res 
Citation: Heruth DP, Zhang LQ, Ye SQ (2011) Nicotinamide Phosphoribosyltransferase in Rheumatoid Arthritis. J Bioanal Biomed 3: 076-080. doi:10.4172/1948-593X.1000047

78: $356-365$.

50. Kim SR, Bae SK, Choi KS, Park SY, Jun HO, et al. (2007) Visfatin promotes angiogenesis by activation of extracellular signal-regulated kinase 1/2. Biochem Biophys Res Commun 357: 150-156.

51. Kim SR, Bae YH, Bae SK, Choi KS, Yoon KH, et al. (2008) Visfatin enhances ICAM-1 and VCAM-1 expression through ROS-dependent NF-kappaB activation in endothelial cells. Biochim Biophys Acta 1783: 886-895.

52. Liu SW, Qiao SB, Yuan JS, Liu DQ (2009) Visfatin stimulates production of monocyte chemotactic protein-1 and interleukin-6 in human vein umbilical endothelial cells. Horm Metab Res 41: 281-286.

53. Bae YH, Bae MK, Kim SR, Lee JH, Wee HJ, et al. (2009) Upregulation of fibroblast growth factor-2 by visfatin that promotes endothelial angiogenesis. Biochem Biophys Res Commun 379: 206-211.

54. Lin H (2007) Nicotinamide adenine dinucleotide: beyond a redox coenzyme Org Biomol Chem 5: 2541-2554.

55. Di Girolamo M, Dani N, Stilla A, Corda D (2005) Physiological relevance of the endogenous mono(ADP-ribosyl)ation of cellular proteins. FEBS J 272: 45654575 .

56. Dantzer F, Ame JC, Schreiber V, Nakamura J, Menissier-de Murcia J, et al. (2006) Poly(ADP-ribose) polymerase-1 activation during DNA damage and repair. Methods Enzymol 409: 493-510.

57. Donmez G, Guarente L (2010) Aging and disease: connections to sirtuins Aging Cell 9: 285-290.
58. Hassa PO, Buerki C, Lombardi C, Imhof R, Hottiger MO (2003) Transcriptional coactivation of nuclear factor-kappaB-dependent gene expression by $\mathrm{p} 300$ is regulated by poly(ADP)-ribose polymerase-1. J Biol Chem 278: 45145-45153.

59. Gonzalez-Rey E, Martínez-Romero R, O’Valle F, Aguilar-Quesada R, Conde $C$, et al. (2007) Therapeutic effect of a poly(ADP-ribose) polymerase-1 inhibitor on experimental arthritis by downregulating inflammation and Th1 response. PLoS One 2: e1071.

60. Fulco M, Cen Y, Zhao P, Hoffman EP, McBurney MW, et al. (2008) Glucose restriction inhibits skeletal myoblast differentiation by activating SIRT1 through AMPK-mediated regulation of Nampt. Dev Cell 14: 661-673.

61. Bitterman KJ, Anderson RM, Cohen HY, Latorre-Esteves M, Sinclair DA (2002) Inhibition of silencing and accelerated aging by nicotinamide, a putative negative regulator of yeast sir2 and human SIRT1. J Biol Chem 277: 4509945107.

62. Lin SJ, Ford E, Haigis M, Liszt G, Guarente L (2004) Calorie restriction extends yeast life span by lowering the level of NADH. Genes Dev 18: 12-16.

63. Dvir-Ginzberg M, Gagarina V, Lee EJ, Booth R, Gabay O, et al. (2011) TNFalpha-mediated cleavage and inactivation of SirT1 in human osteoarthritic chondrocytes. Arthritis Rheum 2011

64. Ungerstedt JS, Blomback M, Soderstrom T (2003) Nicotinamide is a potent inhibitor of proinflammatory cytokines. Clin Exp Immunol 131: 48-52.

65. Van Gool F, Gallí M, Gueydan C, Kruys V, Prevot PP, et al. (2009) Intracellula NAD levels regulate tumor necrosis factor protein synthesis in a sirtuindependent manner. Nat Med Feb 15: 206-210. 